\title{
Which Patients Should Be Administrated Prophylactic Antibacterial Agents? A Study of Bacteriuria or Funguria by Urine Culture Taken from the Renal Pelvis in Children with Ureteropelvic Junction Obstruction
}

\author{
Gao-Yan Deng*, Li-Yu Zhang, Zhong-Ming Li, Ying-Quan Wen \\ Department of Pediatric Surgery, Guang Zhou Women and Children's Medical Center, Guang Zhou, China \\ E-mail: 'denggaoyanemail@163.com \\ Received July 21, 2011; revised August 30, 2011; accepted September 9, 2011
}

\begin{abstract}
Objective: To detect bacteriuria or funguria by urine culture taken from the renal pelvis directly before Anderson-Hynes pyeloplasty. Methods: 290 patients who underwent Anderson-Hynes pyeloplasty for ureteropelvic junction obstruction (UPJO) were included in a retrospective analysis. Urine was obtained directly before the renal pelvis was opened, and was carried to the laboratory for bacterial culture. Clinical features were analyzed to evaluate risk factors for bacteriuria or funguria by comparing patients whose urine yielded positive cultures to those whose urine cultures were negative for bacteria or yeast. Results: Eighteen patients (6.2\%) had positive urine cultures, including six cultures positive for Escherichia coli (E. coli), four for Pseudomonas aeruginosa, three for klebsiella pneumoniae, one for maltophilia monad, one for Enterococcus faecium, one for Candida albicans, one for Candida parapsilosis, and one for yeast not otherwise specified. Bacteriuria or funguria was significantly correlated with four clinical features: fever, urinary urgency, and history of nephrostomy or pyeloplasty. Conclusions: Bacteriuria or funguria was less common in children with UPJO, and the majority of organisms were identified as Escherichia coli, Pseudomonas aeruginosa, or Klebsiella pneumoniae. Prophylactic antibacterial agents were probably necessary in those patients who had signs of urinary tract infection (UTI), or history of nephrostomy or pyeloplasty.
\end{abstract}

Keywords: Bacteriuria, Ureteropelvic Junction Obstruction, Urine Culture, Pyeloplasty.

\section{Introduction}

There have been no published reports concerning bacteriuria or funguria discovered by urine culture taken directly from the renal pelvis, despite the extensive literature on urinary tract infection (UTI) in children with hydronephrosis $[1,2]<$ B5 B6 $></ C>$ In our study, the relationship between clinical features and bacteriuria or funguria was also analyzed to evaluate risk factors for these conditions.

\section{Methods}

\subsection{Patients}

A total of 300 patients (320 kidneys) underwent Anderson-Hynes pyeloplasty between May 1999 and January 2009 in our hospital. Urine was taken directly from the renal pelvis of 270 patients ( 290 kidneys) $[210$ boys and 80 girls, with a median age of 1year (range 1month to 12 years)] for bacterial culture; urine of the remaining 30 cases was not cultured either because the patient had no signs of UTI, or for other reasons. Prophylactic antibacterial agents were administrated to those patients who had signs and symptoms of UTI (fever and urinary urgency) prior to admission, and the surgical procedure was postponed until these signs and symptoms disappeared. A low dose of antibacterial agents was also provided to patients who had a history of nephrostomy. Routinely administer antibiotics was IV just prior to skin incision.

\subsection{Urine Culture Prior to Surgery}

Anderson-Hynes pyeloplasty was performed in all cases. Obtained immediately before the renal pelvis was opened 
with a needle inserted into the pelvis, urine for culture was hand-carried in a sterile tube to the laboratory. These specimens were cultured by inoculation onto cystine lactose electrolyte-deficient agar, and were incubated in an atmosphere containing 5\% carbon dioxide. Cultures were classified according to the criteria in standard use in the laboratory, and bacteriuria or funguria was defined as 10/L or more colony-forming units (CFUs). Antibiotic sensitivity was determined using standard techniques [3]. For our analysis, we classified specimens as resistant or sensitive, although some organ- isms are not routinely tested against certain antibiotics.

\subsection{Recording of Clinical Features}

Clinical features including age, gender, whether ureteropelvic junction obstruction (UPJO) was discovered by prenatal ultrasound, abdominal pain, urgency, previous nephrostomy and pyeloplasty were recorded in the medical history. "Fever" was defined as body temperature higher than $37.5^{\circ}$, "mass" indicated an abdominal or lumbar mass on physical examination, and "hematuria" meant gross hematuria in our study. Ultrasound was performed in all patients, hydronephrotic grade was determined in accordance with the criteria established by the Society of Fetal Urology, [4] and "parenchymal thickness" was the averaged measurements of three different locations(over upper pole, lowest pole and the most dilated calyx).

\subsection{Statistical Analysis}

Sample-size calculations were carried out using Excel software 2003 (Microsoft corporation, Washington D.C., U.S.A.), and statistical analysis was carried out by SPSS 13.0 statistical software (SPSS, Chicago, IL, USA). Some clinical features (gender, whether UPJO was discovered by prenatal ultrasound, fever, abdominal pain, hematuria, urgency, mass, previous nephrostomy, and pyeloplasty) were viewed as univariates, and another features (age, grade of hydronephrosis and parenchymal thickness) were numerical variates. Logistic tests were performed to assess the relationship between the clinical features and bacteriuria or funguria A P-value of $<0.05$ was considered to be statistically significant.

\section{Results}

Eighteen of 300 patients $(6.2 \%)$ had urine cultures positive for bacteria or yeast, including six positive for E. coli, four for pseudomonas aeruginosa, three for klebsiella pneumoniae, one for maltophilia monad, one for Enterococcus faecium, one for Candida albicans, one for Candida parapsilosis, and one for yeast not otherwise specified. Most bacteria were sensitive to cefoperazone, aztreonam, imipenem, vancomycin, and amikacin, whereas the yeast was sensitive to nysfungin and fluconazole (Table 1). No patient with a urine culture positive for E.

Table 1. Antibiotic sensitivity of the positive cases.

\begin{tabular}{|c|c|c|c|c|c|c|c|c|c|}
\hline & Sensitivity & E.coli. & $\mathrm{PA}$ & $\mathrm{KP}$ & MM & $\mathrm{EF}$ & $\mathrm{CA}$ & $\mathrm{CP}$ & yeast \\
\hline cases & & 6 & 4 & 3 & 1 & 1 & 1 & 1 & 1 \\
\hline CFUs $\left(* 10^{7}\right)$ & & $7 \pm 2$ & $8 \pm 3$ & $7 \pm 3$ & 6 & 10 & 10 & 5 & 10 \\
\hline Ampicillin & $8 / 15(53)$ & 4 & 2 & 2 & & & & & \\
\hline Cefazolin & $10 / 15(67)$ & 5 & 3 & 2 & & & & & \\
\hline Cefuroxime & $10 / 15(67)$ & 4 & 3 & 1 & 1 & 1 & & & \\
\hline Ceftriaxone & $12 / 15(80)$ & 5 & 4 & 1 & 1 & 1 & & & \\
\hline Ceftazidime & $11 / 15(73)$ & 5 & 3 & 1 & 1 & 1 & & & \\
\hline Cefepime & $10 / 14(71)$ & 5 & 3 & & 1 & 1 & & & \\
\hline cefoperazone & $10 / 10(100)$ & 5 & 3 & 1 & 1 & & & & \\
\hline Aztreonam & $9 / 9(100)$ & 4 & 3 & 1 & 1 & & & & \\
\hline Imipenem & $12 / 12(100)$ & 6 & 3 & 1 & 1 & 1 & & & \\
\hline Vancomycin & $13 / 13(100)$ & 5 & 3 & 3 & 1 & 1 & & & \\
\hline Gentamicin & $13 / 15(87)$ & 6 & 3 & 2 & 1 & 1 & & & \\
\hline Amikacin & $15 / 15(100)$ & 6 & 4 & 3 & 1 & 1 & & & \\
\hline Cetotaxime & $12 / 15(80)$ & 5 & 3 & 2 & 1 & 1 & & & \\
\hline Tobramycin & $12 / 15(80)$ & 5 & 3 & 2 & 1 & 1 & & & \\
\hline Nitrofurantoin & $10 / 15(67)$ & 4 & 3 & 2 & 1 & & & & \\
\hline Trimethoprim & $12 / 15(80)$ & 5 & 3 & 2 & 1 & 1 & & & \\
\hline Nysfungin & $1 / 3(33)$ & & & & & & 1 & & \\
\hline fluconazole & $2 / 3(67)$ & & & & & & & 1 & 1 \\
\hline
\end{tabular}

$\boldsymbol{\Delta}$ : Values are expressed as number/total (percentage). PA: Pseudomonas aeruginosa; KP: klebsiella pneumoniae; MM: maltophilia monad; EF: Enterococcus faecium; CA: Candida albicans; CP: Candida parapsilosis; CFUs: colony-forming unites. 
coli had a history of nephrostomy or pyeloplasty. In contrast, some patients with cultures positive for other organisms cases had a history of nephrostomy or pyeloplasty $(\mathrm{P}<0.05)$.

The relationship between clinical features and bacteriuria or funguria was also analyzed to assess the risk factors for developing bacteriuria or funguria. Parameter estimation was obtained by maximum likelihood using the Logistic test. bacteriuria or funguria had no signify- cant relationship with some features (age, gender, whether UPJO was discovered by prenatal ultrasound, pain, hematuria, mass, grade of hydronephrosis, and parenchymal thickness). Another four clinical features (fever, urgency, previous nephrostomy history, and history of pyeloplasty) had significant correlation with bacteriuria or funguria (Table 2). These four factors were identified as risk factors for developing bacteriuria or funguria by the omnibus tests of model coefficients using the forward likelihood ratio test (Table 3).

Table 2. Parameter estimation obtained using maximum likelihood test. ※

\begin{tabular}{|c|c|c|c|c|c|}
\hline Variables & negative group & positive group & B & $\operatorname{Exp}(B)$ & $P$ value \\
\hline age(months) & $38 \pm 5$ & $24 \pm 3$ & -0.0034 & 0.9966 & 0.7936 \\
\hline male & 230 & 16 & 0.4477 & 1.5647 & 0.7667 \\
\hline female & 42 & 2 & & & \\
\hline DPU & 127 & 7 & 1.2564 & 3.5129 & 0.3770 \\
\hline fever & 8 & 5 & 3.9694 & 52.9558 & 0.0102 \\
\hline pain & 48 & 1 & -2.8222 & 0.0595 & 0.2026 \\
\hline hematuria & 7 & 0 & -15.3862 & 0.0000 & 0.9992 \\
\hline urgency & 3 & 1 & 3.8935 & 49.0847 & 0.0109 \\
\hline mass & 32 & 2 & 2.1741 & 8.7935 & 0.2091 \\
\hline hydronephrosis grade & & & -0.7773 & 0.4596 & 0.9167 \\
\hline $\mathrm{I}^{\circ} \bullet$ & 0 & 0 & & & \\
\hline $\mathrm{II}^{\circ} \bullet$ & 0 & 0 & & & \\
\hline $\mathrm{IIII}^{\circ} \bullet$ & 125 & 9 & -0.4748 & 0.6220 & 0.9366 \\
\hline $\mathrm{IV}^{\circ} \bullet$ & 82 & 5 & -0.2796 & 0.7560 & 0.9460 \\
\hline $\mathrm{V}^{\circ} \bullet$ & 65 & 4 & -1.5460 & 0.2131 & 0.5072 \\
\hline thickness $(\mathrm{mm})$ & $5.2 \pm 1.1$ & $4.8 \pm 1.2$ & -0.3948 & 0.6738 & 0.6731 \\
\hline Nephrostomy history & 4 & 6 & 6.43555 & 623.6281 & 0.0000 \\
\hline pyeloplasty history & 2 & 5 & 4.9987 & 148.2242 & 0.0004 \\
\hline Constant & & & -5.2693 & 0.0051 & 0.0217 \\
\hline
\end{tabular}

$※$ : Overall percentage: $96.2 \%$; $\mathbf{\sim}$ : Discovered by prenatal ultrasound; $\diamond$ : hydronephrosis grade according to the Society of Fetal Urology criteria [4]; B: partial regression coefficient; $\operatorname{Exp}(\mathrm{B})$ : Odds ratio.

Table 3. Result of likelihood ratio test.

\begin{tabular}{|c|c|c|c|c|c|}
\hline & & Wald Chi-Square & -2 Log Likelihood & Nagelkerke R Square & P value \\
\hline \multirow[t]{2}{*}{ Step $1 ※$} & Step & 24.301 & 110.620 & 0.216 & 0.0000 \\
\hline & Model & 24.301 & & & \\
\hline \multirow[t]{2}{*}{ Step2 $\triangle$} & Step & 24.322 & 86.298 & 0.415 & 0.0000 \\
\hline & Model & 48.624 & & & \\
\hline \multirow[t]{2}{*}{ Step $3 \diamond$} & Step & 19.030 & 67.268 & 0.559 & 0.0000 \\
\hline & Model & 67.654 & & & \\
\hline \multirow[t]{2}{*}{ Step4ロ } & Step & 10.095 & 57.173 & 0.632 & 0.0015 \\
\hline & Model & 77.749 & & & \\
\hline
\end{tabular}

$※$ : urgency, $\triangle$ : previous nephrostomy; $\diamond$ : renal surgical history; $\square$ : fever. 


\section{Discussion}

UPJO is common in children, for which Anderson-Hynes pyeloplasty is the classic surgical treatment $[5,6]<\mathrm{B} 1$ $\mathrm{B} 2></ \mathrm{C}<\mathrm{B} 3 \mathrm{~B} 4><$, and there have been some reports concerning about UTI in patients with UPJO [1,2]. Unlike previous studies, in the present study, urine taken directly from the renal pelvis was cultured. The risk factors for developing bacteriuria or funguria were also been determined by Logistic test.

As has been described in previous reports, [8] our study found that E. coli, pseudomonas aeruginosa, and klebsiella pneumoniae were the most common organisms cultured from urine. No patient with a urine culture positive for E. coli had a history of nephrostomy or pyeloplasty, unlike patients with urine cultures positive for other organisms. No previous study has reported this. As has been reported previously, urinary tract abnormalities increase the incidence of bacteriuria or funguria [7]. Because E. coli has better adhesive and migratory capabilities, translocation to the renal pelvis is more likely for this organism, even in patients with no history of nephrostomy or pyeloplasty. Our study also found that most bacteria were sensitive for cefoperazone, aztreonam, imipenem, vancomycin, and amikacin; however, the conclusion was less persuasive, because some patients had received prophylactic antibacterial agents.

There has been no study of the relationship between age or gender and bacteriuria or funguria in patients with UPJO. Our study found that there was no significant relationship between age or gender and bacteriuria or funguria, perhaps because boys were more likely develop UPJO, [5,6] whereas girls were much more likely to develop a UTI after 1 year [9]. Increasingly, UPJO is discovered by prenatal ultrasound [6], but being diagnosed by prenatal ultrasound was not a risk factor to develop bacteriuria or funguria. In addition, hydronephrosis [5], abdominal pain, hematuria, and mass were not risk factors.

As has been discussed in some studies [1], the degree of hydronephrosis was not a risk factor for developing bacteriuria or funguria in our study. No previous study has reported a relationship between parenchymal thickness and bacteriuria or funguria. We found that parenchymal thickness was not a risk factor, although, deterioration in renal function was associated with exacerbation of hydronephrosis and decrease in parenchymal thickness in some patients [5,6]. Prophylactic antibacterial agents were probably necessary in those patients who had signs of urinary tract infection (UTI), or history of nephrostomy or pyeloplasty.

This retrospective study has some limitations. Firstly, prophylactic antibacterial agents had been administrated to those patients who had a nephrostomy catheter or had signs/symptoms of UTI (fever and urgency) prior to the operation. Some patients with negative urine cultures had had signs/symptoms of UTI (Table 2). Thus, some important information might have been lost [2]. In addition, one of the main important factors responsible for resistance or sensitivity of uropathogens is antibiotic pretreatment and the use of prophylactic antibacterial agents [10]. In this study, five patients $(27 \%)$ in the bacteriuria/funguria group and eight patients $(2.9 \%)$ in the culture-negative group had received antibiotics before the urine was cultured. The antibiotic sensitivity results were less reliable when urine culture was not performed prior to administration of prophylactic antibacterial agents, This issue is a topic for future.

\section{Conclusions}

In the present study, we found that four factors: fever, urgency, and history of nephrostomy or pyeloplasty, were significantly correlated with bacteriuria or funguria. Fever and urgency were signs of UTI, and were thus indications for urine culture. History of nephrostomy or renal surgical history increased the risk of bacteriuria or funguria contamination. These four factors had high positive predictive value for positive urine cultures (overall percentage: $96.2 \%$, Table 2). In conclusion, Prophylactic antibacterial agents were probably necessary in those patients who had signs of urinary tract infection (UTI), or history of nephrostomy or pyeloplasty.

\section{References}

[1] C. C. Roth, J. M. Hubanks, B. C. Bright, et al., "Occurrence of Urinary Tract Infection in Children with Significant Upper Urinary Tract Obstruction," Urology, Vol. 73, No. 1, 2009, pp. 74-78. doi:10.1016/j.urology.2008.05.021

[2] S. H. Song, S. B. Lee, Y. S. Park and K. S. Kim, "Is Antibiotic Prophylaxis Necessary in Infants with Obstructive Hydronephrosis?" Journal of Urology, Vol. 177, 2007, pp. 1098-1101.

[3] A. W. Bauer, W. M. Kirby, J. C. Sherris and M. Turck. "Antibiotic Susceptibility Testing by a Standardized Single Disk Method," American Journal of Clinical Pathology, Vol. 45, 1996, pp. 493-496.

[4] C. Delaned, "Antenatal Hydronephrosis: Trends and Management," Urological Nursing, Vol. 25, No. 3, 2005, pp. 179-183.

[5] C. Mami, A. Paolata, A. Palmara, et al., "Outcome and Management of Isolated Moderate Renal Pelvis Dilatation Detected at Postnatal Screening," Pediatric Nephrology, Vol. 24, No. 10, 2009, pp. 2005-2008.

[6] B. Chertin, A. Pollack, D. Koulikov, et al., "Conservative 
Treatment of Ureteropelvic Junction Obstruction in Children with Antenatal Diagnosis of Hydronephrosis: Lessens Learned after 16 Years of Follow-up," European Urology, Vol. 49, 2006, pp. 734-739.

[7] J. J. Zorc, D. A. Kiddoo and N. Kathy, "Shaw: Diagnosis and Management of Pediatric Urinary Tract Infections," Clinical Microbiology Reviewa, Vol. 18, No. 2, 2005, pp. 417-422. doi:10.1128/CMR.18.2.417-422.2005

[8] J. H. Lee, H. S. Choi, J. K. Kim, et al., "Nonrefluxing Neonatal Hydronephrosis and the Risk of Urinary Tract
Infection," Journal of Urology, Vol. 179, No. 4, 2008, pp. 1524-1528.

[9] A. Hellstrom, E. Hanson, S. Hansson, K. Hjalmas, et al., "Associatin between Urinary Symptoms at 7 Years Old and Previous Urinary Tract Infection," Archives of Disease in Childhood, Vol. 66, No. 2, 1991, pp. 323-334.

[10] S. A. Lutter, M. L. Currie, L. B. Mitz, et al., "Antibiotic Resistance Patterns in Children Hospitalized for Urinary Tract Infection," Archives of Pediatrics \& Adolescent Medicine, Vol. 159, 2005, pp. 924-927. 